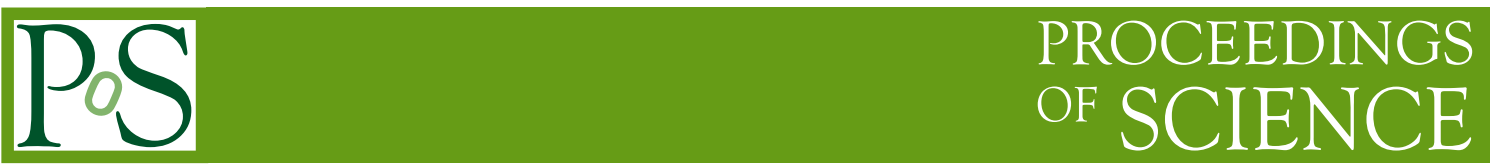

\title{
Latest Results from the Daya Bay Experiment
}

\author{
J.P. Ochoa-Ricoux ${ }^{* \dagger}$ \\ Instituto de Física, Pontificia Universidad Católica de Chile, Santiago, Chile \\ E-mail: jpochoa@uc.cl
}

The Daya Bay Reactor Neutrino Experiment consists of eight functionally identical detectors placed underground at different baselines from six $2.9 \mathrm{GW}_{\text {th }}$ nuclear reactors. With a growing dataset that constitutes the largest sample of reactor antineutrino interactions ever collected to date, Daya Bay is able to study a wide range of topics of interest in neutrino physics. In this letter I review the latest results from Daya Bay on different fronts, such as the latest measurement of the oscillation parameters that drive the disappearance of electron antineutrinos at short baselines, and a search for light sterile neutrino mixing, among others. I also describe a recent measurement of the evolution of the reactor antineutrino flux and spectrum over multiple fuel cycles in 1230 days. A $3.1 \sigma$ discrepancy in the antineutrino flux evolution with recent predictions is observed, indicating an overestimation of the predicted antineutrino flux from ${ }^{235} \mathrm{U}$ and suggesting that this fission isotope could be the primary contributor to the reactor antineutrino anomaly.

The 19th International Workshop on Neutrinos from Accelerators-NUFACT2017

25-30 September, 2017

Uppsala University, Uppsala, Sweden

* Speaker.

${ }^{\dagger}$ for the Daya Bay Collaboration. 


\section{The Daya Bay Reactor Neutrino Experiment}

The Daya Bay experiment is a collaboration of approximately 230 scientists from China, the United States, Taiwan, Hong Kong, the Czech Republic, Russia, and Chile. It is located in the Daya Bay nuclear power complex, about $55 \mathrm{~km}$ northeast of Hong Kong, on the outskirts of the city of Shenzhen, China. With a total of six $2.9 \mathrm{GW}_{\text {th }}$ reactor cores, this plant ranks among the most powerful nuclear stations in the world.

Daya Bay was designed to make a precise measurement of the $\theta_{13}$ neutrino mixing angle by sampling the abundant reactor electron antineutrino flux in two kinds of locations, one where the oscillation effects are very small (near), and another where the oscillation effects modulated by $\sin ^{2} 2 \theta_{13}$ are maximal (far). A comparison of the rate and spectral shape of reactor antineutrinos in the two locations allows to cancel many systematic uncertainties to first order and to extract the oscillation parameters with high precision. A schematic describing the experiment can be seen on the left of Fig. 1. Due to the spread of the reactors, Daya Bay was designed with two near sites. A total of eight identically designed detectors are distributed amongst three experimental sites, with two in each near site and four in the far site.
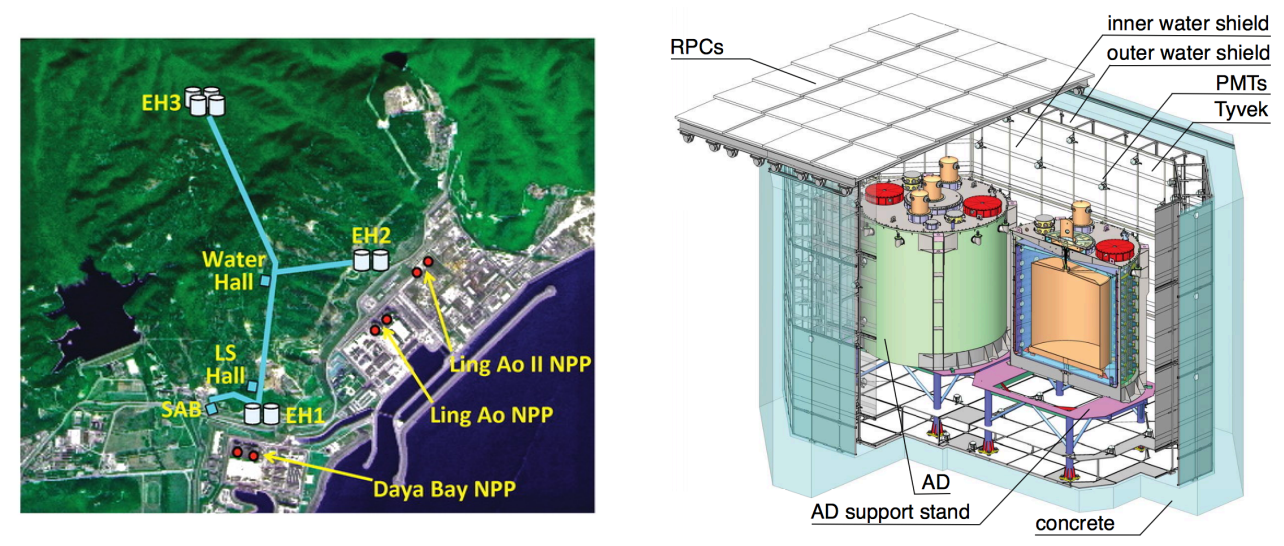

Figure 1: (Left) Schematic layout of the Daya Bay Experiment. A total of eight identically designed antineutrino detectors, represented as cylinders, are deployed in the three experimental halls (EHs). The six reactor cores are represented by the red dots. (Right) Cross-section of all detector systems in a near site. A cross-section of one of the two ADs is shown to illustrate the three nested volumes described in the text.

Antineutrinos are detected via the inverse $\beta$-decay (IBD) reaction, given by: $\bar{v}_{e}+p \rightarrow e^{+}+n$. The antineutrino detectors (ADs) consist of three nested cylindrical volumes separated by concentric acrylic vessels. From outermost to innermost, these volumes contain mineral oil, liquidscintillator (LS) and Gadolinium-doped LS. The light produced by the positrons' loss of energy and annihilation is seen by 192 photomultiplier tubes (PMTs) in each AD. The neutrons are subsequently captured by gadolinium nuclei in the central volume, yielding a clear $\sim 8 \mathrm{MeV}$ light signature. The coincidence of the prompt positron light with that from the delayed neutron capture, separated by time constant of $\sim 30 \mu$ s, allows to drastically suppress the backgrounds.

As shown on the right panel of Fig. 1, the detectors are submerged in instrumented water pools that serve a double purpose: (i) to shield the detectors from ambient radioactivity and cosmogenic 
neutrons, as well as (ii) to tag cosmic-ray muons. Additionally, a retractable roof of four layers of Resistive Plate Chambers (RPCs) covers the pools, providing additional efficiency for muon tagging.

\section{Oscillation Results}

A new measurement of the oscillation parameters that drive electron antineutrino disappearance at short baselines, based on 1230 days of exposure, was recently released [1]. The results are based on a sample amounting to more than 2.5 million electron antineutrino $\left(\bar{v}_{e}\right)$ interactions selected via neutron capture on Gadolinium ( $\mathrm{nGd}$ ), comprising the largest library of reactor antineutrinos ever collected. These results benefit from significant improvements in background reduction and energy calibration. In particular, two of the three Am-C sources in each AD in the Far hall were removed during the 2012 summer installation period, reducing the average correlated Am-C background rate in the far hall by a factor of 4 . Moreover, improvements in calibration allowed to reduce the relative energy scale uncertainty to $<0.2 \%$, resulting in an unprecedentedly small relative detection efficiency of $0.13 \%$.

The spectrum of $\bar{v}_{e}$ 's in the far hall can be seen on the left panel of Fig. 2. The backgrounds amount to less than $2 \%$ in all halls, and are dominated by accidental events, i.e. two uncorrelated signals occasionally satisfying the antineutrino selection criteria. The expectation in the case of no oscillations is shown in blue, indicating a clear deficit caused by oscillations. A relative comparison of the rate and spectral shape of $\bar{v}_{e}$ 's in all halls allows to simultaneously extract the oscillation parameters, yielding $\sin ^{2} 2 \theta_{13}=0.0841 \pm 0.0027$ (stat.) \pm 0.0019 (syst.) and $\left|\Delta m_{32}^{2}\right|=(2.45 \pm 0.06$ (stat.) \pm 0.06 (syst.) $) \times 10^{-3} \mathrm{eV}^{2}$ for the normal hierarchy, or $\left|\Delta m_{32}^{2}\right|=$ $(-2.56 \pm 0.06$ (stat.) \pm 0.06 (syst.) $) \times 10^{-3} \mathrm{eV}^{2}$ for the inverted hierarchy. The results can be equivalently expressed in terms of the effective mass splitting $\Delta m_{\mathrm{ee}}^{2}$ for electron antineutrino disappearance, obtained by combining the $\Delta m_{32}^{2}$ and $\Delta m_{31}^{2}$ terms in the oscillation probability into a single one, yielding $\left|\Delta m_{\text {ee }}^{2}\right|=(2.50 \pm 0.06$ (stat. $) \pm 0.06$ (syst. $\left.)\right) \times 10^{-3} \mathrm{eV}^{2}$ [1]. The confidence regions for $\sin ^{2} 2 \theta_{13}$ and $\Delta m_{\mathrm{ee}}^{2}$ are shown on the right side of Fig. 2. The fit is in excellent agreement with the expectation from three-flavor neutrino oscillations, with $\chi^{2} / \mathrm{ndf}=234.7 / 263$. These results currently constitute the world's most precise estimates of these two parameters, with the uncertainty in the mass splitting being comparable to that achieved in accelerator experiments [2].

A measurement of $\bar{v}_{e}$ disappearance has also been performed using an IBD sample tagged via neutron capture on Hydrogen $(\mathrm{nH})$ [3]. Not only is the IBD $\mathrm{nH}$ sample completely distinct from the $\mathrm{nGd}$ one, but the systematic uncertainties are very different, making this measurement a truly independent determination of $\theta_{13}$. A comparison of the relative $\bar{v}_{e}$ rates in the Daya Bay detectors with a dataset based on an exposure of 621 days yields $\sin ^{2} 2 \theta_{13}=0.071 \pm 0.011$ with $\chi^{2} / \mathrm{ndf}=6.3 / 6$. An improved $\mathrm{nH}$ analysis using the spectral shape in addition to the rate, as well as a significantly larger dataset, is being prepared.

\section{Search for Light Sterile Neutrino Mixing}

Daya Bay is also able to cover a large amount of ground in the search for light sterile neutrino mixing. In the minimal extension to the three-flavor neutrino model, where only one sterile neu- 

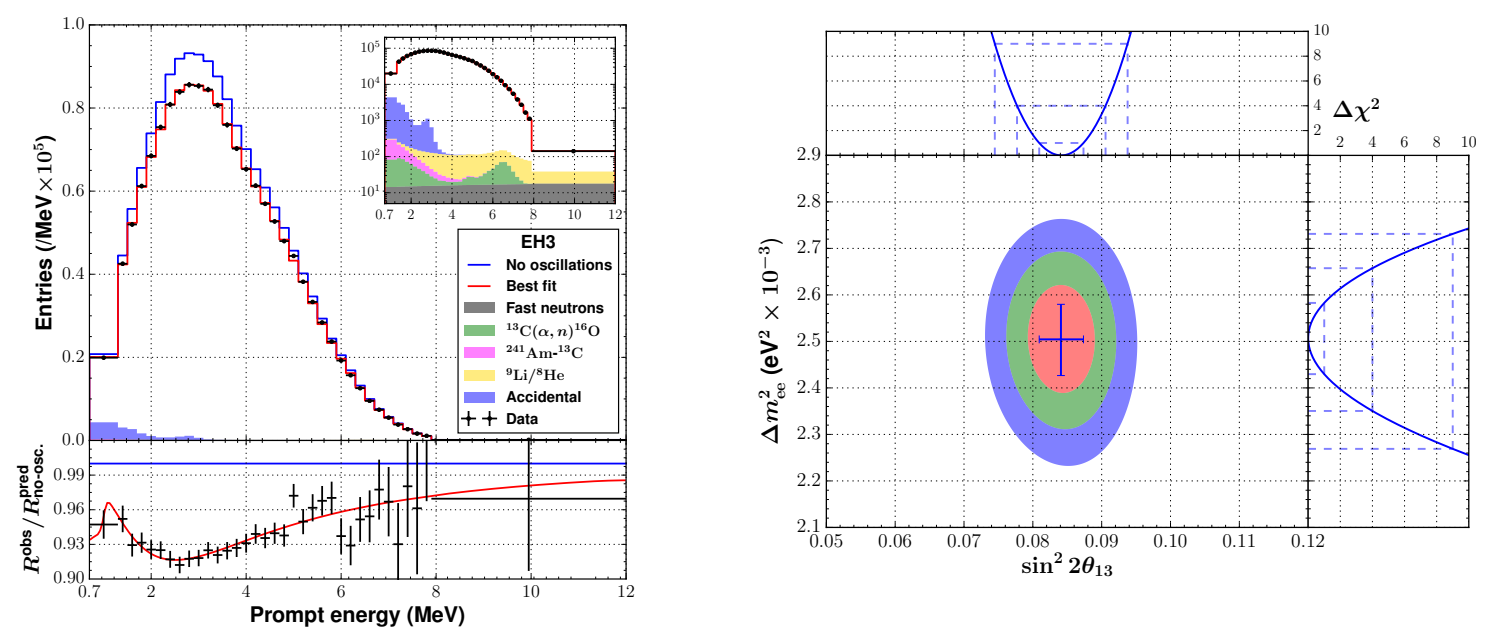

Figure 2: (Left) Reconstructed positron energy spectrum for the $\bar{v}_{e}$ candidate interactions in the Far hall. The measurements are compared with the prediction assuming no oscillation (blue line) and the best-fit three-flavor neutrino oscillation model (red line). (Right) Confidence regions for $\sin ^{2} 2 \theta_{13}$ and the effective mass-splitting $\left|\Delta m_{\mathrm{ee}}^{2}\right|$.

trino is added to the three active ones, the search essentially amounts to looking for an additional spectrum distortion with a frequency $\Delta m_{41}^{2}$ different to $\Delta m_{e e}^{2}$. No deviation from the three-flavor framework is found in the latest analysis, performed with a dataset collected over 621 days [4]. The resulting exclusion limits are shown on the left side of Fig. 3. Daya Bay's unique configuration of multiple baselines from three groups of nuclear reactors to three experimental sites allows to cover a range of $\Delta m_{41}^{2}$ spanning over three orders of magnitude. These limits provide the world's most stringent constraints in the $\left|\Delta m_{41}^{2}\right| \lesssim 0.2 \mathrm{eV}^{2}$ region and represent an improvement of roughly a factor of two over the previous publication [5].

This measurement can be used to probe the hints for electron antineutrino appearance observed by the LSND [6] and MiniBooNE [7] experiments if combined with $\stackrel{(-)}{v_{\mu}}$ disappearance searches. In the minimal extension to the three-flavor model, the oscillation probability of $\stackrel{(-)}{v_{\mu}} \rightarrow \stackrel{(-)}{v_{e}}$ is roughly proportional to $\sin ^{2} 2 \theta_{\mu e}=4\left|U_{e 4}\right|^{2}\left|U_{\mu 4}\right|^{2}$. The quantities $\left|U_{e 4}\right|^{2}$ and $\left|U_{\mu 4}\right|^{2}$ can be independently constrained with $\stackrel{(-)}{v_{e}}$ and $\stackrel{(-)}{\nu}_{\mu}$ disappearance searches respectively, and the results combined to constrain $\sin ^{2} 2 \theta_{\mu e}$. Such a combination has recently been released by the Daya Bay and MINOS collaborations based on their results as well as those from Bugey-3 [8]. The three experiments are sensitive to complementary regions of parameter space, providing the limits on $\sin ^{2} 2 \theta_{\mu e}$ shown on the right panel of Fig. 3. These limits span over six orders of magnitude in $\Delta m_{41}^{2}$ and exclude the parameter space allowed by LSND and MiniBooNE for $\Delta m_{41}^{2}<0.8 \mathrm{eV}^{2}$ at $95 \%$ C.L.

\section{Comparisons with Reactor Model}

Daya Bay also recently released an improved, high-statistics measurement of the absolute reactor antineutrino flux and spectral shape using a data set acquired over 621 days [9]. The ratio of the measured IBD yield to the expectation from the Huber-Mueller model $[10,11]$ yields $R_{\mathrm{DYB}}=$ 

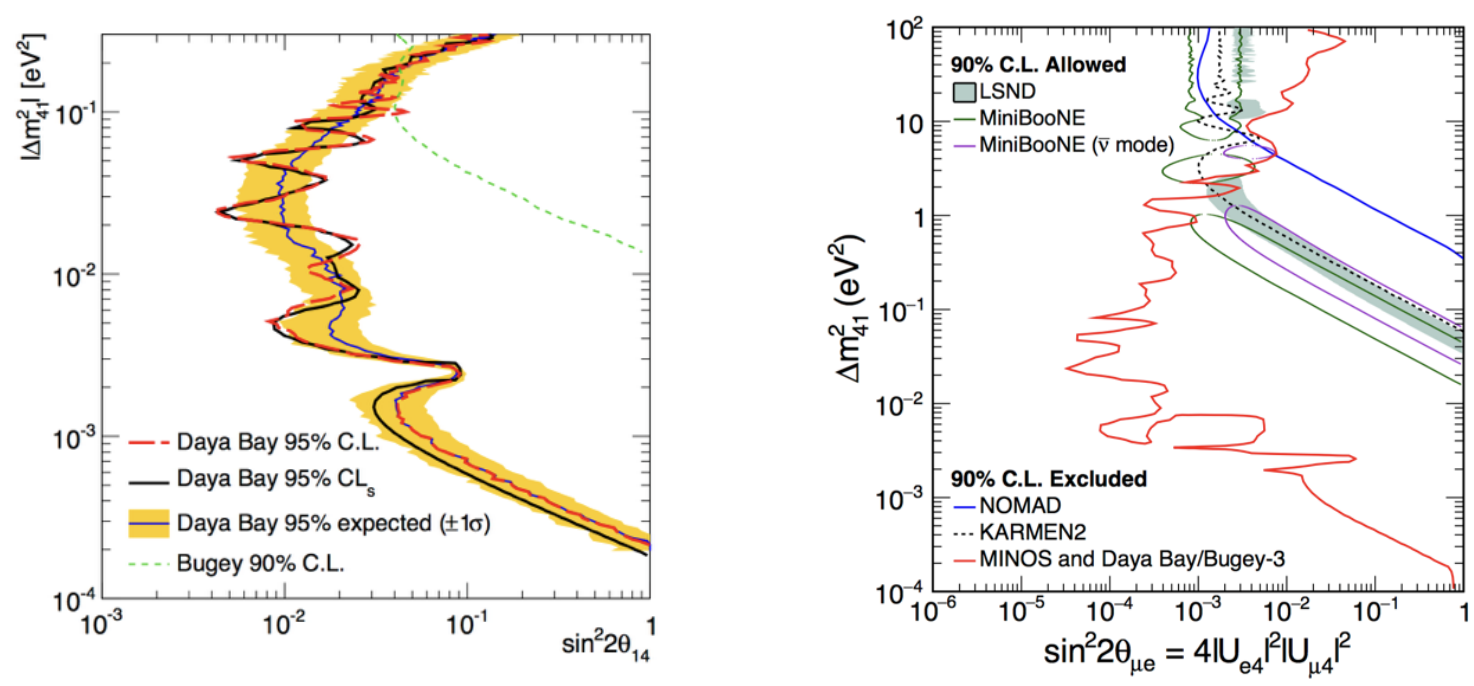

Figure 3: (Left) Exclusion contours for the DYB-only analysis in the $\left(\sin ^{2} 2 \theta_{41},\left|\Delta m_{41}^{2}\right|\right)$ parameter space. Two independent and complementary statistical methods, Feldman-Cousins and $\mathrm{CL}_{\mathrm{s}}$, are used to set the exclusion limits. (Right) MINOS and Daya Bay + Bugey-3 combined $90 \%$ exclusion limit obtained using the $\mathrm{CL}_{\mathrm{s}}$ method. The regions allowed by LSND and MiniBooNE are also shown.

$0.946 \pm 0.020$ (exp.). This result is in agreement with past short baseline reactor experiments, whose average is $R_{\text {global }}^{\text {past }}=0.942 \pm 0.009$ (exp.), and thus confirms the so-called reactor antineutrino anomaly [12]. In addition, the comparison of the predicted and measured absolute reactor spectrum shape reveals some discrepancies with the Huber-Mueller model, as shown on the left side of Fig. 4. In particular, there is a significant excess of events in the 4-6 MeV range. These events have all the IBD characteristics, are power-correlated, and are time-independent. The feature does not appear in the ${ }^{12} \mathrm{~B}$ spectra, disfavoring any detector effects.

A novel measurement that studies how the yield and spectral shape of reactor antineutrinos changes as a fuel cycle progresses sheds additional light on these discrepancies [13]. The analysis is done in terms of the effective fission fraction $F_{239}$, defined as the percentage of total fissions from the ${ }^{239} \mathrm{Pu}$ isotope as seen by each AD. The right of Fig. 4 shows $F_{239}$ vs. time, and how this quantity increases in a fuel cycle as the corresponding quantity for ${ }^{235} \mathrm{U}, F_{235}$, decreases. The left of Fig. 5 shows how the evolution of the total IBD yield as a function of $F_{239}$ is different from the expectation in the Huber-Mueller model at the $3.1 \sigma$ level. From these data it is possible to extract information on the individual IBD yields for the major fissioning isotopes $\left({ }^{235} \mathrm{U}\right.$ and $\left.{ }^{239} \mathrm{Pu}\right)$ while making conservative assumptions on the minor ones $\left({ }^{241} \mathrm{Pu}\right.$ and $\left.{ }^{238} \mathrm{U}\right)$. The results are shown as the shaded areas in the right of Fig. 5. The individual yield for ${ }^{239} \mathrm{Pu}$ is in good agreement with the expectation, but there is a significant tension in the ${ }^{235} \mathrm{U}$ case. The hypothesis that all isotopes contribute equally to the reactor antineutrino anomaly is disfavored at the $2.8 \sigma$ level, weakening the sterile neutrino interpretation of this anomaly. Together, the flux, spectral shape, and fuel evolution discrepancies strongly suggest that unaccounted systematics, or other problems with the prediction, are at lest partially at play. 

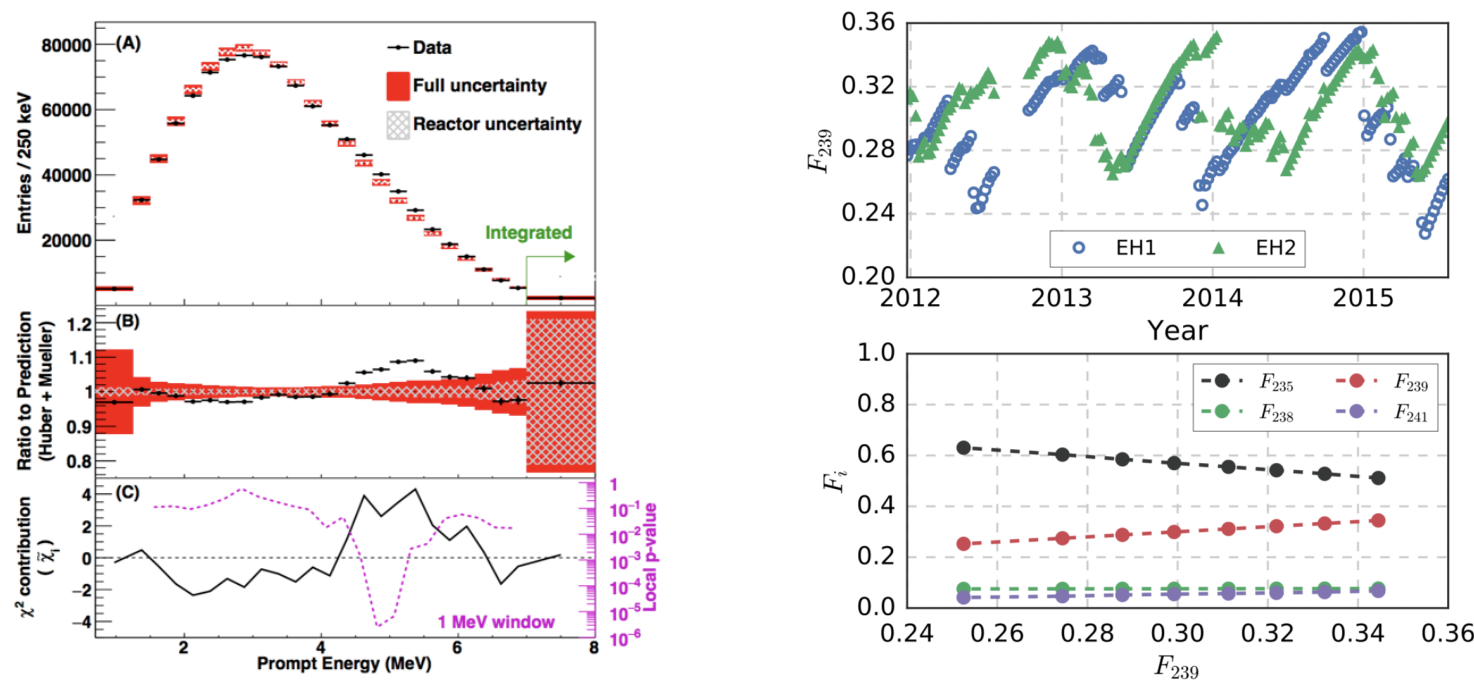

Figure 4: (Left) Comparison of predicted and measured prompt energy spectra of nGd events. The prediction is based on the Huber-Mueller model and is normalized to the number of measured events. The ratio of the measured prompt energy spectrum to the predicted spectrum, as well as the $\chi^{2}$ distribution of each bin (black solid curve) and the local p-values for $1 \mathrm{MeV}$ energy windows (magenta dashed curve), are shown in the lower panels. (Right) The top panel shows $F_{239}$ for the EH1 and EH2 ADs, as calculated weekly from reactor data. The bottom panel shows the effective fission fractions for the four main isotopes as a function of $F_{239}$.
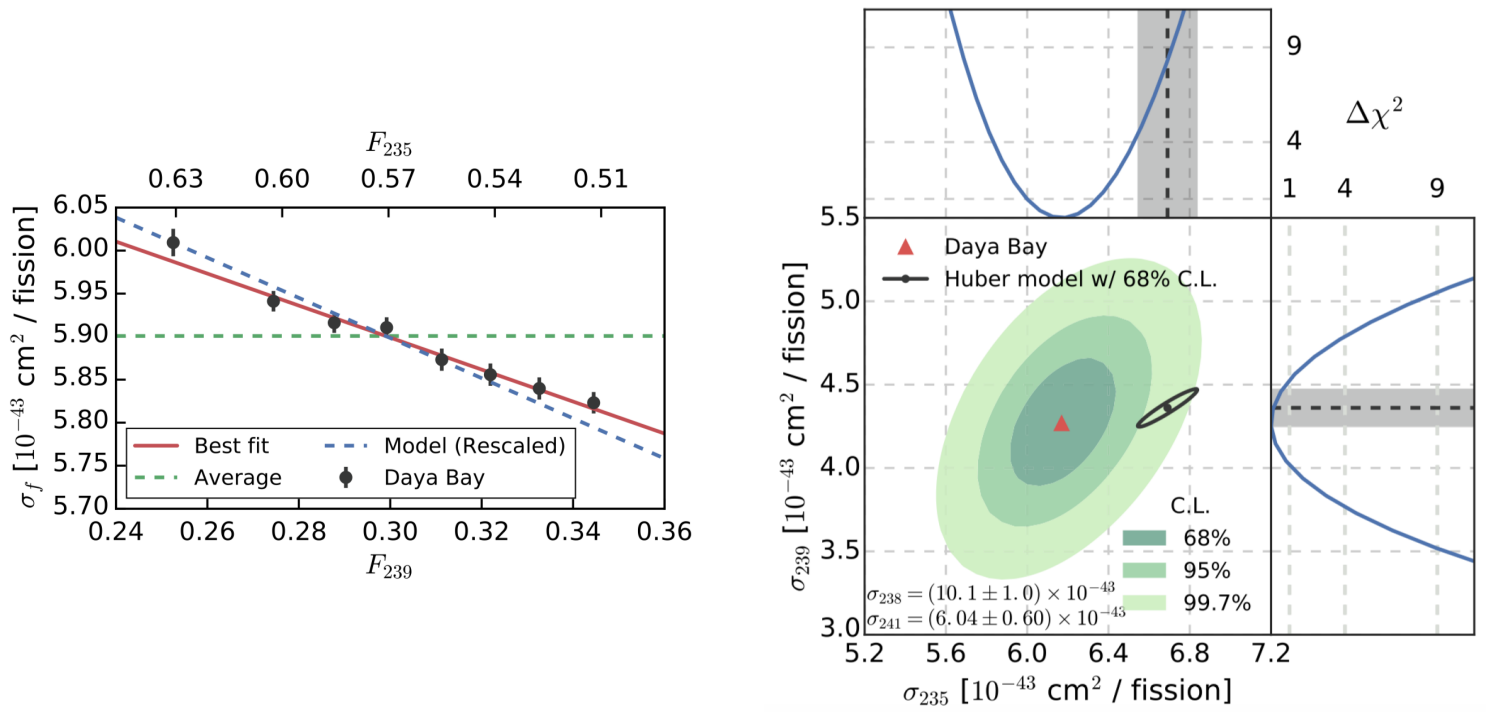

Figure 5: (Left) Measured and predicted total IBD yield per fission $\sigma_{f}$ vs. $F_{239}$. (Right) Allowed regions for the combined measurement of the individual ${ }^{239} \mathrm{Pu}$ and ${ }^{235} \mathrm{U}$ yields $\left(\sigma_{239}\right.$ and $\sigma_{235}$ respectively). The $68 \%$ C.L. allowed region for the Huber-Mueller expectation is shown in black.

\section{Summary and Outlook}

Daya Bay recently released an updated measurement of the $\theta_{13}$ and $\Delta m_{e e}^{2}$ parameters with unprecedented precision, as well an independent determination of $\theta_{13}$ via neutron capture on hydrogen, and an improved search for light sterile neutrino mixing. The discrepancies between the 
observed and predicted reactor flux and spectral shape are seen in Daya Bay with unprecedentedly high-statistics, and a new analysis studying the evolution of these quantities within a fuel cycle has revealed that the ${ }^{235} \mathrm{U}$ isotope could be the primary contributor to the reactor antineutrino anomaly. Other results not covered here have also been recently released, such as a study of wave-packet treatment of neutrino oscillation [14] and an analysis of the correlation between the seasonal variation in the cosmic muon flux and the atmospheric temperature [15]. The Daya Bay experiment is expected to run until 2020, and work is ongoing to further reduce systematic errors through the installation of a FADC readout system in one of the near ADs and through the execution of a special calibration campaign, among other activities. The primary goal is to reduce the uncertainties in $\theta_{13}$ and $\Delta m_{e e}^{2}$ below $3 \%$.

\section{References}

[1] F.P. An et al. (Daya Bay). Measurement of electron antineutrino oscillation based on 1230 days of operation of the Daya Bay experiment. Phys. Rev., D95(7):072006, 2017.

[2] K. A. Olive et al. Review of Particle Physics. Chin. Phys., C38:090001, 2014.

[3] F.P. An et al. (Daya Bay). New measurement of $\theta_{13}$ via neutron capture on hydrogen at Daya Bay. Phys. Rev., D93(7):072011, 2016.

[4] F.P. An et al. (Daya Bay). Improved Search for a Light Sterile Neutrino with the Full Configuration of the Daya Bay Experiment. Phys. Rev. Lett., 117(15):151802, 2016.

[5] F.P. An et al. (Daya Bay). Search for a Light Sterile Neutrino at Daya Bay. Phys.Rev.Lett., 113:141802, 2014.

[6] A.A. Aguilar-Arevalo et al. (LSND). Evidence for neutrino oscillations from the observation of anti-neutrino(electron) appearance in a anti-neutrino(muon) beam. Phys. Rev., D64:112007, 2001.

[7] A. A. Aguilar-Arevalo et al. (MiniBooNE). Improved Search for $\bar{v}_{\mu} \rightarrow \bar{v}_{e}$ Oscillations in the MiniBooNE Experiment. Phys. Rev. Lett., 110:161801, 2013.

[8] P. Adamson et al. (MINOS and Daya Bay). Limits on Active to Sterile Neutrino Oscillations from Disappearance Searches in the MINOS, Daya Bay, and Bugey-3 Experiments. Phys. Rev. Lett., 117(15):151801, 2016. [Addendum: Phys. Rev. Lett.117,no.20,209901(2016)].

[9] F.P. An et al. (Daya Bay). Improved Measurement of the Reactor Antineutrino Flux and Spectrum at Daya Bay. Chin. Phys., C41(1):013002, 2017.

[10] P. Huber. On the determination of anti-neutrino spectra from nuclear reactors. Phys. Rev. C, 84:024617, 2011.

[11] T. Mueller et al. Improved Predictions of Reactor Antineutrino Spectra. Phys. Rev. C, 83:054615, 2011.

[12] G. Mention et al. The Reactor Antineutrino Anomaly. Phys.Rev., D83:073006, 2011.

[13] F.P. An et al. (Daya Bay). Evolution of the Reactor Antineutrino Flux and Spectrum at Daya Bay. Phys. Rev. Lett., 118(25):251801, 2017.

[14] F.P. An et al. (Daya Bay). Study of the wave packet treatment of neutrino oscillation at Daya Bay. Eur. Phys. J., C77(9):606, 2017.

[15] F.P. An et al. (Daya Bay). Seasonal Variation of the Underground Cosmic Muon Flux Observed at Daya Bay. JCAP, 1801(01):001, 2018. 